\title{
Saddlepoints in P-Pivot Classes of Skew Matrices*
}

\author{
Marjorie L. Stein \\ Institute for Basic Standards, National Bureau of Standards, Washington, D.C. 20234
}

(September 13, 1974)

\begin{abstract}
A finite class of skew matrices can be associated via "principal pivots" with a self-dual linear system. Given a row index $h$, it is known that there is a skew matrix in the class with nonnegative $h$ th row. Using this "saddlepoint theorem," we prove a similar result for nonpositive rows. An open question is whether such a class of skew matrices contains one with both a nonnegative row and a nonpositive row. We show that this "double saddlepoint property" holds for arbitrarily large degenerate cases and for all skew matrices of small order.
\end{abstract}

Key words: Combinatorial equivalence; linear inequalities; linear programs; pivot operations; skewsymmetry.

A finite class of skew matrices can be associated with a self-dual linear system. It is known $[1,2]^{1}$ that each such class contains one or more skew matrices with one type of "saddlepoint," a sign configuration consisting of a nonnegative row and corresponding nonpositive column. Using this saddlepoint theorem, we prove a similar result with the reverse sign pattern.

An open question is whether such a class of matrices contains a skew matrix that simultaneously exhibits both a nonnegative row and a nonpositive row. We can prove that this "double saddlepoint" property does hold for skew matrices of small order and for arbitrarily large finite degenerate cases, but the general problem remains unsolved.

\section{P-Pivots and the First Saddlepoint Theorem}

Dual systems of linear equations can be represented in the following tableau format of Tucker [5]:
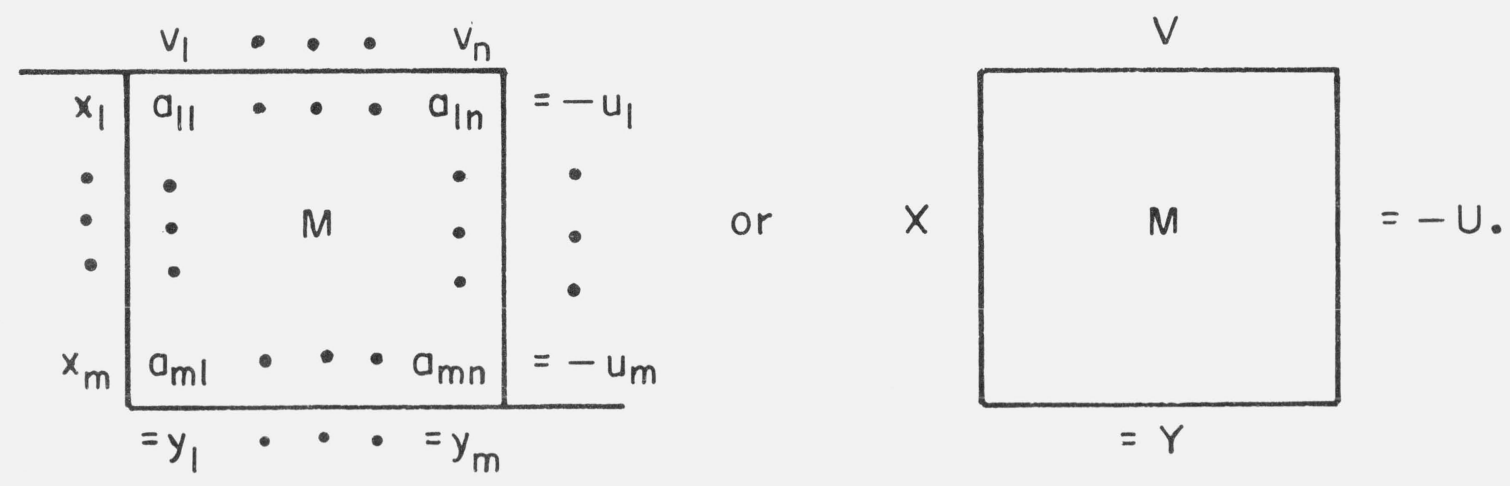

\footnotetext{
AMS Subject Classification: $15 \mathrm{~A} 39,15 \mathrm{~A} 21$.
* This work was completed while the author was a National Academy of Sciences-National Research Council Postdoctoral Research Associate at the National Bureau of Standards, Washington, D.C. This paper is dedicated to Professor A. W. Tucker on the occasion of his retirement from Princeton Iniversity.

${ }^{1}$ Figures in brackets indicate the literature references at the end of this paper.
} 
Here $X, Y, U^{T}$ and $V^{T}$ are row vectors. The tableau simultaneously represents the column system $X M=Y$ and the row system $M V=-U$. The systems are always dual in the sense of orthogonality, for

$$
X U+Y V=X(-M V)+(X M) V=0 .
$$

Since this paper is concerned with the sign patterns in matrices, we necessarily allow variables and coefficients to take values only in an ordered field. The symbols $\oplus$ and $\ominus$ respectively denote nonnegative and nonpositive elements.

Definition 1: An $n \times n$ matrix $A$ is skew when $A=-A^{T}$.

Throughout this paper $A$ denotes a skew matrix of order $n$. Over an ordered field the diagonal entries of $A$ must be zero. The tableau of $A$ is written as follows:

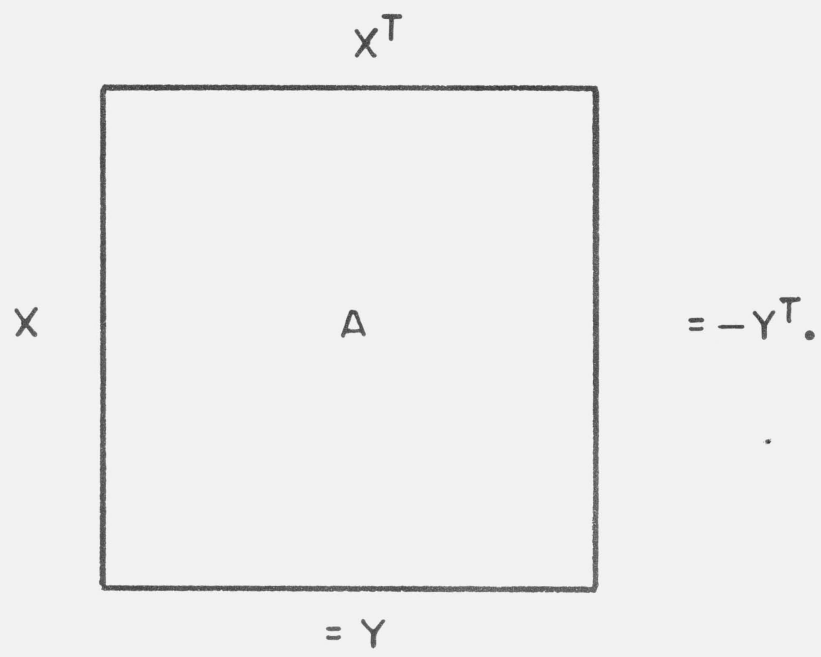

By negative transposition it is clear that any solution $(X, Y)$ to the column system $X A=Y$ is also a row solution $(Y, X)$ to $A X^{T}=-Y^{T}$ and conversely. Hence each of the linear systems is self-dual because $X$ and $Y$ must be orthogonal.

Definition 2: A principal pair pivot or $P$-pivot in (the tableau of) a self-dual linear system is a simultaneous exchange of variables $x_{i}$ with $y_{i}$ and $x_{j}$ with $y_{j}$, provided $a_{i j}=-a_{j i} \neq 0$, while leaving the remaining variables fixed.

The $P$-pivot involves solving the $i$ th column (row) equation for $x_{j}$ and the $j$ th equation for $x_{i}$, substituting the resulting expressions into the remaining equations. At the same time there occurs a (principal) permutation that exchanges row $i$ with row $j$ and column $i$ with column $j$ while fixing the remaining rows and columns.

We say that we $P$-pivot on $a_{i j}, a_{j i}$ in the matrix $A$. For $a_{i j} \neq 0$, this operation yields another skew matrix $\bar{A}$ with the self-dual solution $(\bar{X}, \bar{Y})$ to $\bar{X} \bar{A}=\bar{Y}$ such that $\bar{x}_{i}=y_{i}, \bar{x}_{j}=y_{j}, \bar{y}_{i}=x_{i}, \bar{y}_{j}=x_{j}$ and for all $k \neq i, j$ we have $\bar{x}_{k}=x_{k}, \bar{y}_{k}=y_{k}$. The new. skew matrix $\bar{A}$ is given by the following entries (recall that $i \neq j$ ):

$$
\begin{aligned}
& \bar{a}_{i j}=-a_{i j}^{-1} . \\
& \bar{a}_{i h}=-a_{i j}^{-1} a_{j h} \quad \text { for } h \neq i, j . \\
& \bar{a}_{k j}=a_{k i} a_{i j}^{-1} \quad \text { for } k \neq i, j . \\
& \bar{a}_{k h}=a_{k h}-a_{i j}^{-1}\left(a_{i h} a_{k j}-a_{k i} a_{j h}\right) \quad \text { for } k \neq i, j \text { and } h \neq i, j . \\
& \bar{a}_{t t}=0 \quad \text { for all } t .
\end{aligned}
$$


Checking the above formulae requires only straightforward algebraic manipulations.

Schematically, a $P$-pivot or sequence of $P$-pivots corresponds to inversion of a nonsingular principal submatrix of $A$ and the appropriate extensions to the rest of $A$ :
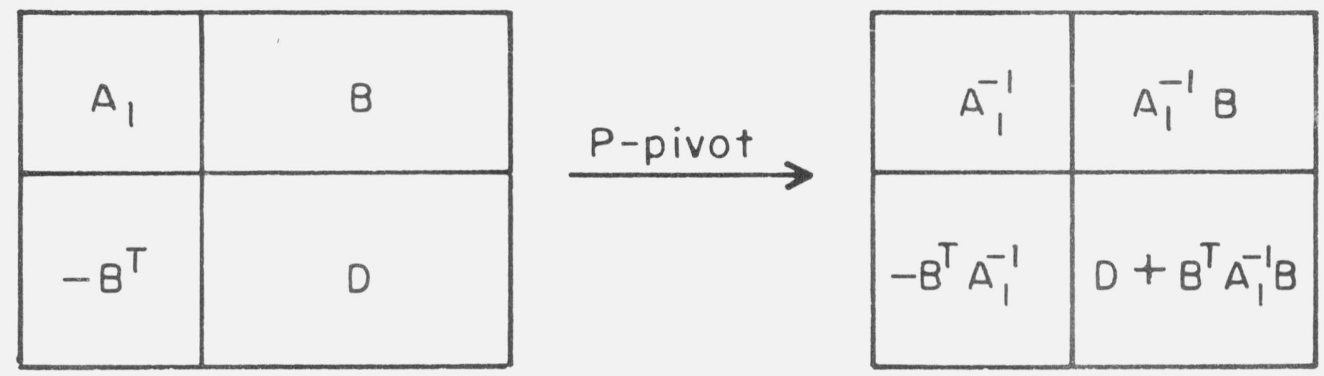

A principal permutation places the nonsingular submatrix $A_{1}$ in the upper corner.

Example: In this $4 \times 4$ self-dual tableau we show a $P$-pivot on the starred entries:
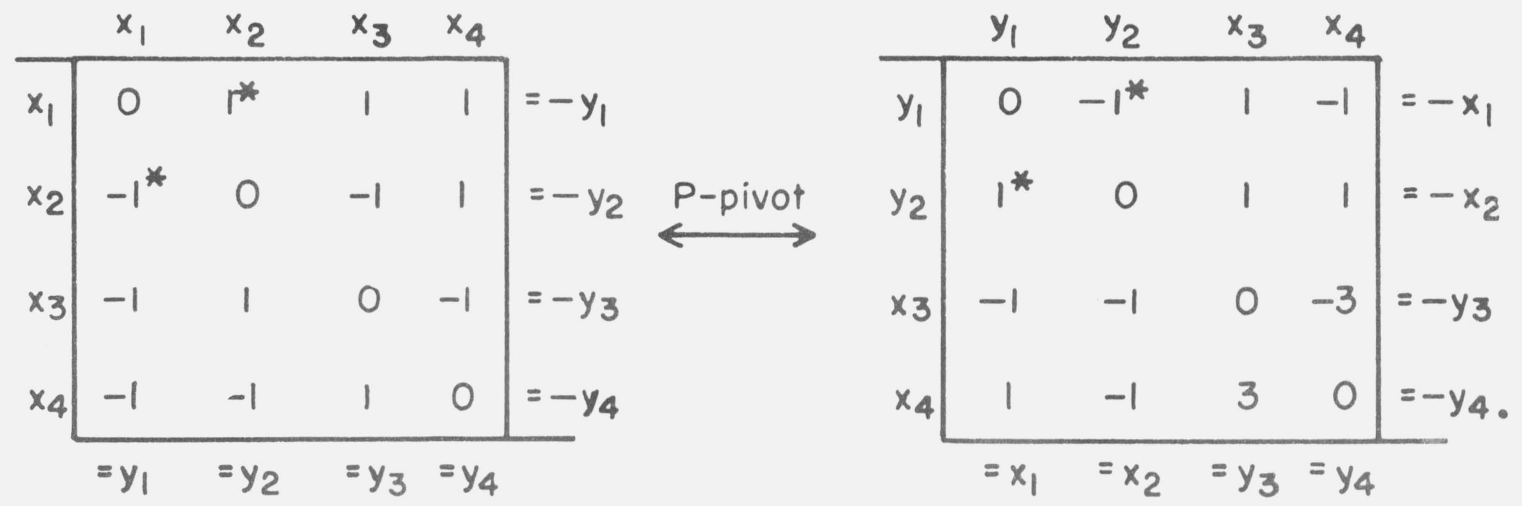

Sample solution: $(X, Y)=\left(x_{1}, x_{2}, x_{3}, x_{4}, y_{1}, y_{2}, y_{3}, y_{4}\right)=(1,0,-1,0,1,0,1,2)$. $(\bar{X}, \bar{Y})=\left(y_{1}, y_{2}, x_{3}, x_{4}, x_{1}, x_{2}, y_{3}, y_{4}\right)=\left(\bar{x}_{1}, \ldots, \bar{x}_{4}, \bar{y}_{1}, \ldots, \bar{y}_{4}\right)$.

$P$-pivots preserve both skewsymmetry and solution sets. The new tableau has the same solution set as the original one in the sense that values assigned to the variables in one tableau will hold for the same variables in the other one even though their positions may have changed.

From a given skew matrix of order $n$, only a finite number of skew matrices can be obtained by a sequence of $P$-pivots [1, Theorem 2.2; 2, Theorem 2]. Such matrices are in fact a subset of Tucker's "combinatorial equivalence class" [4] for the given matrix, and they are called P-pivot transforms.

Definition 3: A nontrivial solution $(X, Y)$ of $X A=Y$ is elementary if the only other nontrivial solutions having the same zero components as $(X, Y)$ are of the form $(k X, k Y)$ for scalars $k \neq 0$.

Definition 4: A zero saddlepoint of type (1) in a matrix is a sign configuration consisting of a nonnegative row and a nonpositive column.

Theorem 1: (First Saddlepoint Theorem) Given a skew matrix A of order $\mathrm{n}$ and an index $\mathrm{h}$, $\mathrm{l} \leqslant \mathrm{h} \leqslant \mathrm{n}$, there exists a nonnegative elementary solution of $\mathrm{XA}=\mathrm{Y}$ such that $\mathrm{x}_{\mathrm{h}}+\mathrm{y}_{\mathrm{h}}>0$, and there exists a skew matrix $\overline{\mathrm{A}}$ which is a P-pivot transform of $\mathrm{A}$ and such that $\overline{\mathrm{a}}_{\mathrm{hj}} \geqslant 0$ for all $\mathrm{j}, 1 \leqslant \mathrm{j} \leqslant \mathrm{n}$.

Theorem 1 says the existence of a type (1) saddlepoint occurs simultaneously with a non- 
negative elementary solution and gives the theorem its name. Since the proof of Theorem 1 is presented elsewhere in full detail [1, Theorem 4.2; 2 , Theorem 3], we omit it here.

\section{The Second Saddlepoint Theorem}

A result related to Theorem 1 is the following [1, Theorem 3.1]:

Theorem 2: Let A be a skew matrix of order n. By a finite sequence of P-pivots outside the first row and column of $\mathrm{A}$ we can obtain a skew matrix $\overline{\mathrm{A}}$ with either a nonnegative first row or a nonnegative row whose first column entry is positive. Schematically, we obtain one of the following:
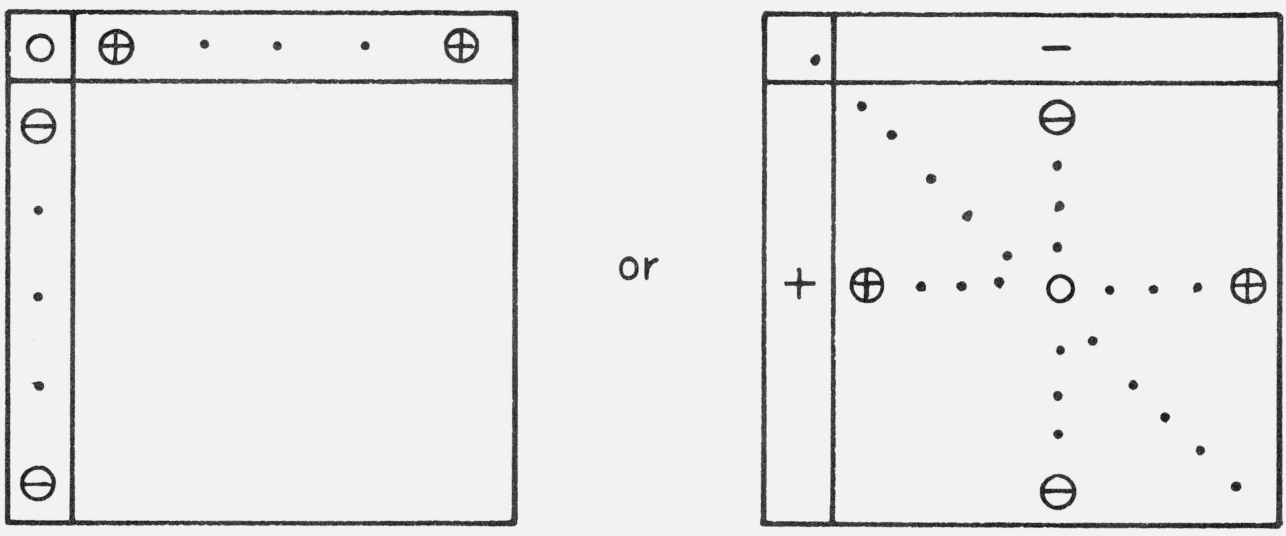

Theorem 2 can be generalized to $P$-pivots outside any given row and the corresponding column. Hence the first row and column can be considered the "distinguished" ones without loss of generality.

DeFINITION 5: A zero saddlepoint of type (2) in a matrix is a sign configuration consisting of a nonpositive row and a nonnegative column.

THEOREM 3: Let $\mathrm{A}$ be a skew matrix of order $\mathrm{n}$ and let $\mathrm{h}$ be an index, $\mathrm{l} \leqslant \mathrm{h} \leqslant \mathrm{n}$. By a finite sequence of $\mathrm{P}$-pivots outside the $\mathrm{h}$ th row and column of $\mathrm{A}$ we can obtain a skew matrix $\overline{\mathrm{A}}$ with either a nonpositive $\mathrm{h}$ th row or a row in which the $\mathrm{h}$ th column entry is negative and every other entry is nonnegative.

Schematically, we obtain one of the following for $h=1$ :
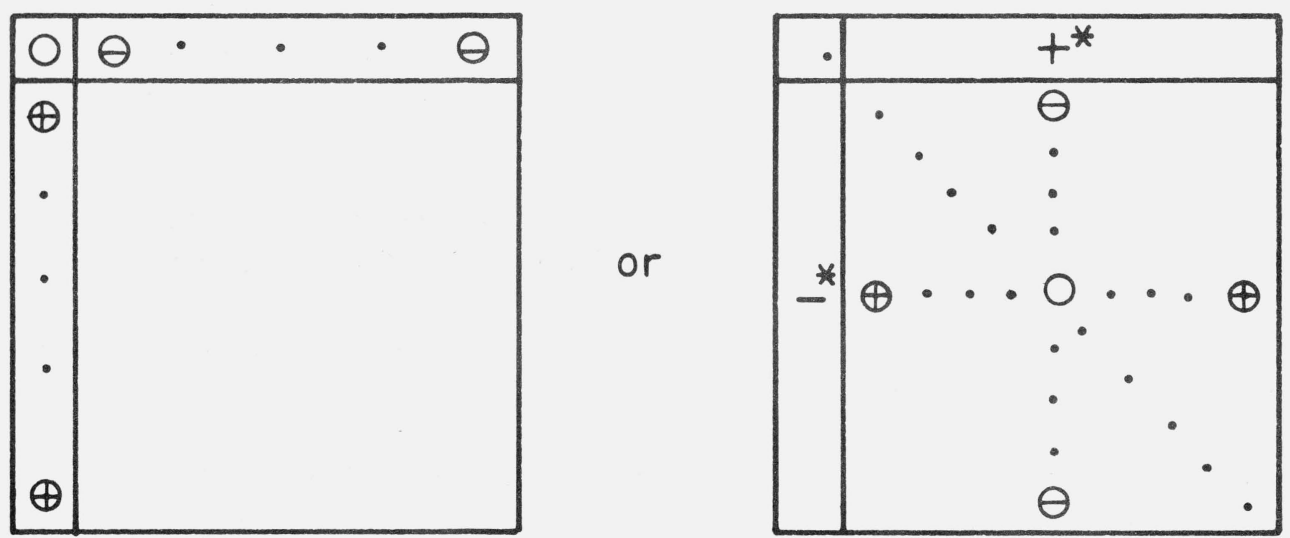

A $P$-pivot on the starred entries in the second alternative yields a saddlepoint of type (2) in the distinguished row and column. 
Proof: Given the skew matrix $A$, form the skew matrix $B$ by multiplying the distinguished row and corresponding column of $A$ by -1 , leaving the rest of $A$ unchanged. A $P$-pivot on $a_{i j}, a_{j i}$ outside the distinguished row and column of $A$ corresponds to a $P$-pivot on $b_{i j}, b_{j i}$ in $B$. From the formulae following Definition 2 we can easily obtain the entries of the $P$-pivot transform $\bar{B}$ in terms of the corresponding entries of $\bar{A}$. When $h$ is the index for the distinguished row and column, $a_{h t}=-b_{h t}$ and $a_{t h}=-b_{t h}$ for all $t, 1 \leqslant t \leqslant n$. Otherwise $a_{k t}=b_{k t}$. Hence $\bar{B}=\bar{A}$ except for the distinguished row and column, where $\bar{b}_{h t}=-\bar{a}_{h t}$ and $\bar{b}_{t h}=-\bar{a}_{t h}$ for all $t$.

Thus the same sequence of $P$-pivots outside a distinguished row and column in $A$ that produces a saddlepoint of type (1) will produce a saddlepoint of type (2) in the corresponding matrix $B$ and vice versa. Equivalently, we may observe that the following diagram is "commutative":

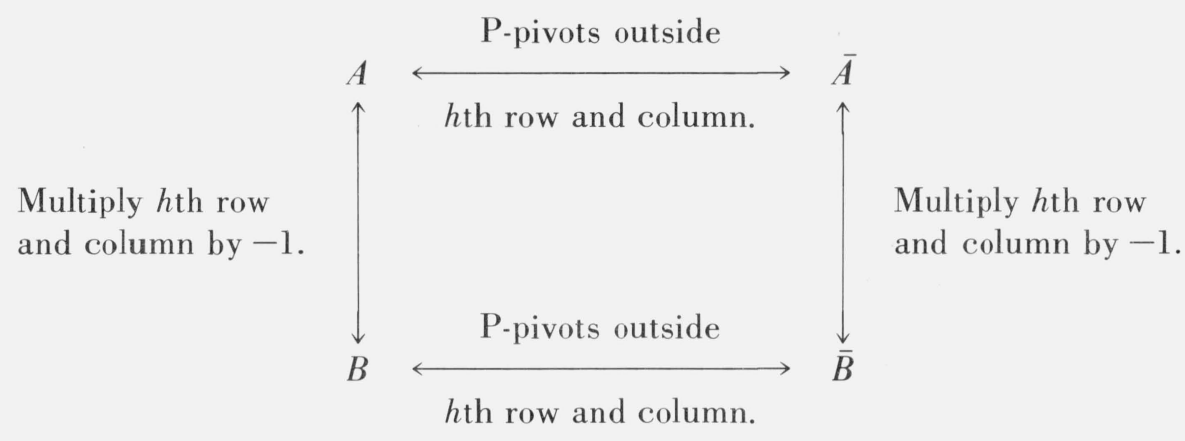

(Same pivot choices as above.)

Combining these observations with the general form of Theorem 2 proves the theorem.

To obtain an analog of Theorem 1 we need to obtain a type of solution whose existence is equivalent to having a type (2) saddlepoint.

Definition 6: A onenegative solution in a self-dual tableau is a solution $(X, Y)$ to $X A=Y$ having precisely one negative component.

Definition 7: A complementary solution in a self-dual tableau is a solution $(X, Y)$ to $X \boldsymbol{A}=Y$ such that $x_{i} y_{i}=0$ for each $i, 1 \leqslant i \leqslant n$.

REMARK: A onenegative complementary solution $(X, Y)$ to $X A=Y$ is of the form:

$$
\begin{aligned}
& x_{j}+y_{j}<0 \quad \text { for one index } j . \\
& x_{i}+y_{i} \geqslant 0 \quad \text { for } i \neq j, 1 \leqslant i \leqslant n . \\
& x_{j} y_{i}=0 \quad \text { for each } i, 1 \leqslant i \leqslant n .
\end{aligned}
$$

Multiplying the $h$ th row and column entries in the matrix of a self-dual tableau by -1 changes the sign of the left-hand sides of the $h$ th row and column equations and of the coefficients of $x_{h}$ in the remaining equations. Hence the original system may be preserved by simultaneously changing the signs of $y_{h}$ and $x_{h}$. Thus any nonnegative complementary solution associated with $A$ would become onenegative when associated with the corresponding $B$; and conversely a onenegative complementary solution with $x_{h}+y_{h}<0$ would become nonnegative. With the additional observation that any nonnegative solution of $\mathrm{XA}=\mathrm{Y}$ must be complementary, we have proved the following result. ${ }^{2}$

${ }^{2}$ I am indebted to Dr. A. J. Goldman for suggesting this method of proof. 
Theorem 4: (Second Saddlepoint Theorem) Given a skew matrix A of order $\mathrm{n}$ and an index $\mathrm{h}$, $1 \leqslant \mathrm{~h} \leqslant \mathrm{n}$, there exists a onenegative complementary solution of $\mathrm{XA}=\mathrm{Y}$ such that $\mathrm{x}_{\mathrm{h}}+\mathrm{y}_{\mathrm{h}}<0$, and there exists a skew matrix $\overline{\mathrm{A}}$ which is a $\mathrm{P}$-pivot transform of $\mathrm{A}$ and such that $\overline{\mathrm{a}}_{\mathrm{hj}} \leqslant 0$ for all $\mathrm{j}, 1 \leqslant \mathrm{j} \leqslant \mathrm{n}$.

Theorem 4 can also be proved independently in a fashion similar to a proof of Theorem 1 [2]. The First and Second Saddlepoint Theorems are logically equivalent.

\section{A Double Saddlepoint Problem}

One question that naturally arises is whether any skew $A$ has a $P$-pivot transform $\bar{A}$ having saddlepoints of both types (1) and (2). One may even ask whether every skew $A$ has a $P$-pivot transform with a "double saddlepoint."

Conjecture: If $A$ is a skew matrix of order $n$, then there exists a $P$-pivot $\operatorname{transform} \bar{A}$ of $A$ having both a nonnegative row and a nonpositive row.

A single row of zeros will satisfy the conjecture. It is proved below for arbitrarily large degenerate cases (Theorem 5) and in general for $n \leqslant 5$ (Theorem 6).

THEOREM 5: If a skew matrix A of order $\mathrm{n}$ can be transformed by a finite number of $\mathrm{P}$-pivots into a skew matrix with a saddlepoint of type (1) having more than one zero entry in the nonnegative row, then A satisfies the Conjecture.

Proof: By Theorem 1, we can always obtain a $P$-pivot transform with a nonnegative row from a given skew matrix $A$ of order $n$. If the nonnegative row is all zero, there is nothing to prove. Hence assume the nonnegative row has at least two zero entries and at least one positive entry. By a principal permutation we obtain:

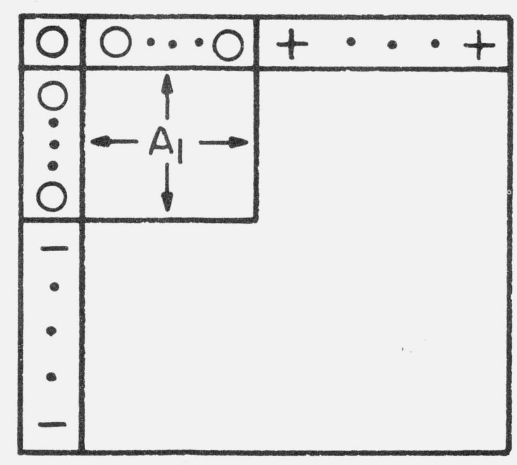

where $A_{1}$ is a nonempty $k \times k$ principal skew submatrix of $A, 1 \leqslant k<n$. (The inverse permutation can always be applied later.) By Theorem 4 we can reach a saddlepoint of type (2) in $A_{1}$ by a finite number of $P$-pivots in $A_{1}$ (i.e., $P$-pivots in $A$ with pivot entries chosen only from $A_{1}$ ). Noting that $P$ pivots in $A_{1}$ do not affect the first row or column of $A$, we obtain: 


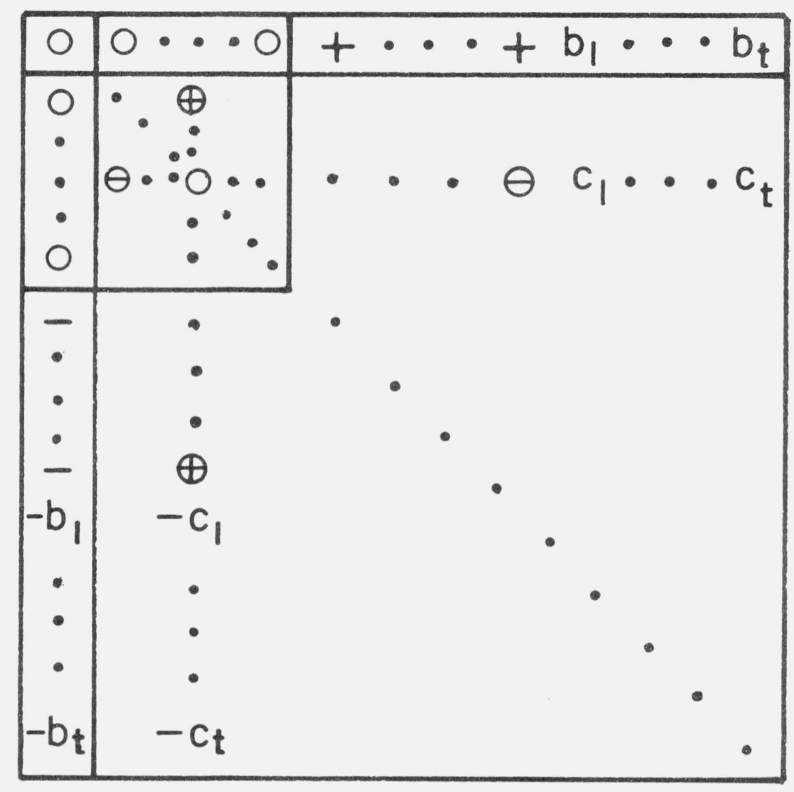

where $b_{\mathrm{i}}, c_{\mathrm{i}}>0$ for $1 \leqslant i \leqslant t$. If the collection of $b$ 's and $c$ 's is empty, there is nothing left to do. Otherwise $P$-pivot on $b_{j},-b_{j}$ where $c_{j} / b_{j}=\max _{i}\left(c_{i} / b_{i}\right)$ to reach the desired form.

To prove the Conjecture for skew matrices of order $n \leqslant 5$, two lemmas will be helpful.

LEMMA 1: If a skew matrix $\mathrm{A}$ of order $\mathrm{n}>1$ can be transformed by a finite number of $\mathrm{P}$-pivots into a skew matrix with a nontrivial nonnegative row, and if some positive entry $\mathrm{a}_{\mathrm{ij}}$ in that row is distinguished by being the only positive entry in its column, then A satisfies the Conjecture.

Proof: $P$-pivot in the nonnegative row and nonpositive column on the distinguished positive and negative entries $a_{i j}, a_{j i}$ to reach the desired form.

Lemma 2: Suppose a skew matrix A of order $\mathrm{n}>1$ can be transformed by a finite number of $\mathrm{P}$ pivots into a skew matrix with a nontrivial nonnegative ith row containing $\mathrm{a}_{\mathrm{ij}}>0$. If there exists another entry $\mathrm{a}_{\mathrm{kj}}$ in the $\mathrm{jth}$ column which, except for $\mathrm{a}_{\mathrm{ij}}$, is the sole positive entry in the $\mathrm{kth}$ row and jth column, then A satisfies the Conjecture.

Proof: $P$-pivot on $a_{i j}, a_{j i}$ to reach the desired form.

Theorem 6: If $\mathrm{A}$ is a skew matrix of order $\mathrm{n} \leqslant 5$, then $\mathrm{A}$ has a $\mathrm{P}$-pivot transform $\overline{\mathrm{A}}$ with both a nonnegative row and a nonpositive row.

Proof: For $n=1$ and $n=2$ the theorem is clearly true since the only possibilities are 0 and

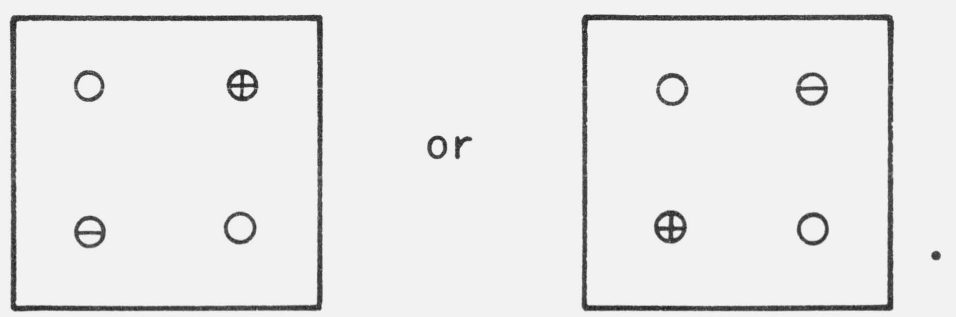

By Theorem 1, we need consider only those cases for $n=3$ where we already have a nonnegative row. The two possibilities are: 


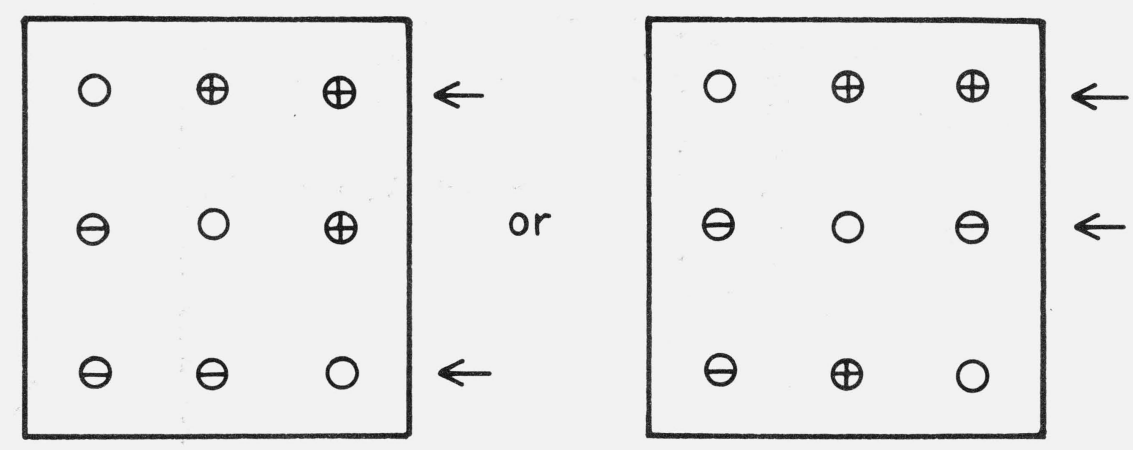

with arrows indicating the desired rows.

For $n=4$ we apply Theorems 1 and 5 , leaving only the eight cases having a nonnegative row with only one zero entry:

(1)

$$
\begin{array}{llll}
0 & + & + & + \\
- & 0 & \oplus & \oplus \\
- & \theta & 0 & \oplus \\
- & \theta & \theta & 0
\end{array} \leftarrow^{(2)}
$$

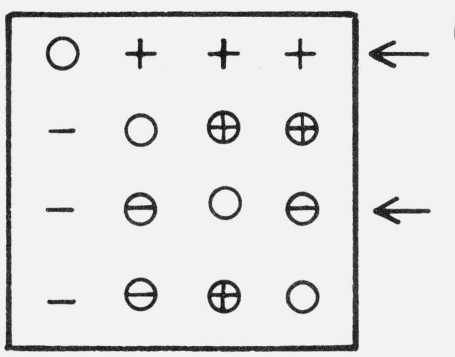

(4)

$$
\begin{array}{cccc}
0 & + & + & + \\
- & 0 & \oplus & - \\
- & \theta & 0 & - \\
- & + & 0
\end{array} \mid \leftarrow
$$

(7)

$$
\begin{array}{rrrr}
0 & + & + & + \\
- & 0 & - & + \\
- & + & 0 & - \\
- & - & + & 0 \\
\hline
\end{array}
$$

(5)

(8)

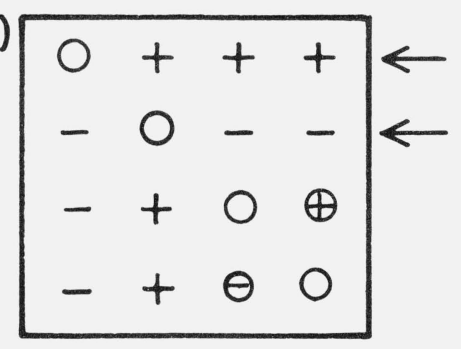

(6)

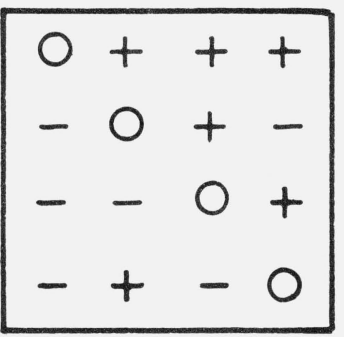

(3)

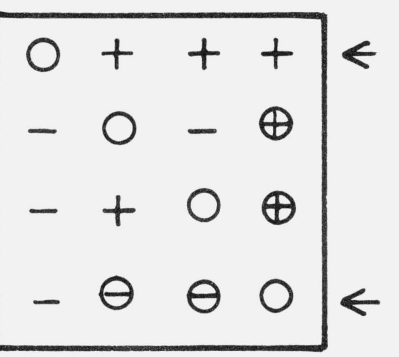

The first five cases and case (8) already possess the desired form, and the remaining cases (6) and (7) satisfy Lemma 2.

For $n=5$, application of Theorems 1 and 5 again limit the cases to be considered. Lemma 1 eliminates other possibilities, narrowing the choices to the following nine sign patterns: 
(1)

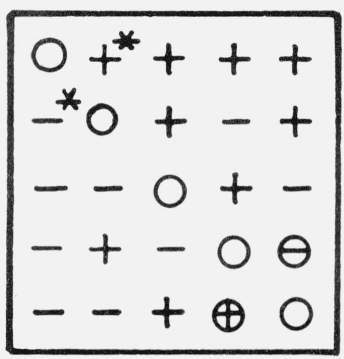

(4)

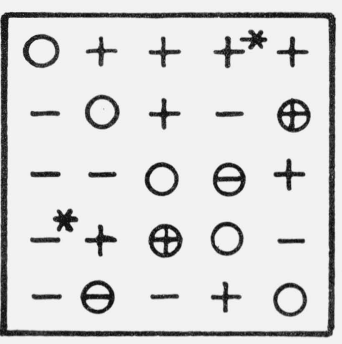

(7)

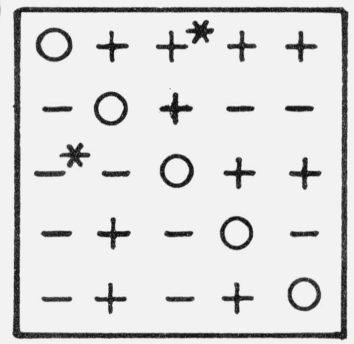

(2)

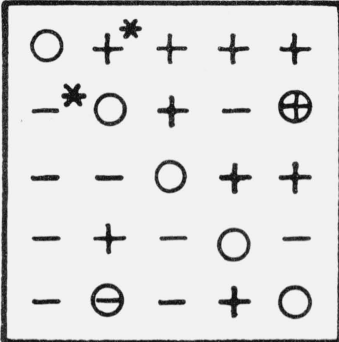

(5)

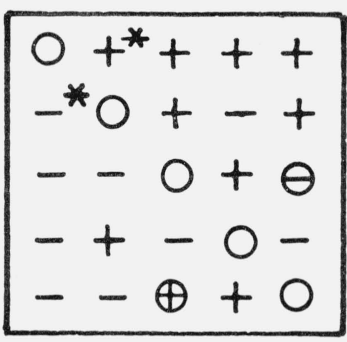

(8)

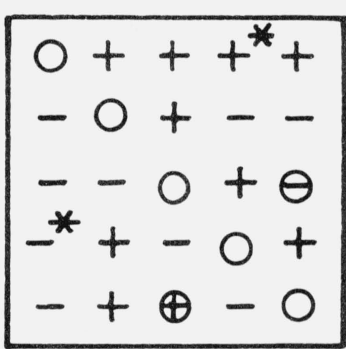

(3)

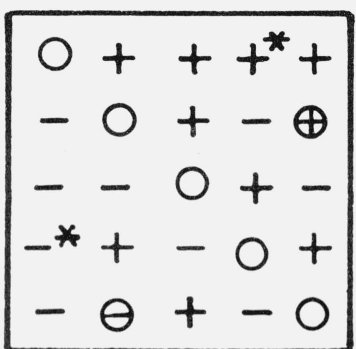

(6)

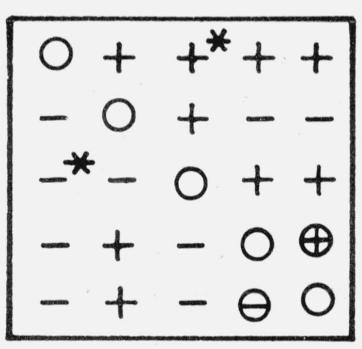

(9)

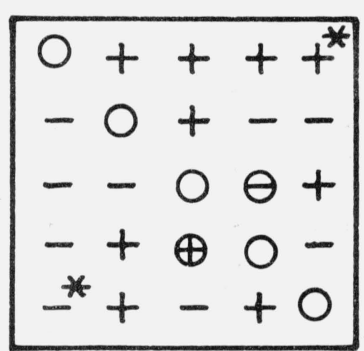

By Lemma 2,P-pivots on the starred entries will yield a double saddlepoint in each case.

\section{Applications}

The First and Second Saddlepoint Theorems have many applications in the theory of dual linear systems and related topics. Both theorems are logically equivalent to Tucker's Skew-Symmetric Matrix Theorem [1, Theorem 5.1; 2, Corollary 1]. From this result the main theorems of linear programming can be elegantly derived [1, Chapter 8]. Other applications include the theory of matrix games [1, Chapter 6] and classical theorems of Gordan, Stiemke, Farkas and von Neumann on linear inequalities [1, Chapter 9].

In view of these applications of the First and Second Saddlepoint Theorems, their generalization to the Double Saddlepoint Conjecture can be expected to have interesting consequences. One consequence of the proved degenerate case of the Conjecture is presented below. Although the Conjecture is still unproved in the general nondegenerate case, a recent undergraduate project at Princeton under the direction of Tucker has examined the $P$-pivot classes of 100 randomlygenerated skew matrices of orders 6 to 10 and has yielded no counterexample. ${ }^{3}$

Theorem 5, which shows the existence of a double saddlepoint for skew matrices if one of the single saddlepoints has an off-diagonal zero, will now be shown to have a surprising consequence for simple pivots in rectangular matrices.

${ }^{3}$ Personal communication from Professor Tucker. 
DeFinition 8: Let $\mathrm{M}$ be an $\mathrm{m} \times \mathrm{n}$ matrix over an ordered field with the tableau

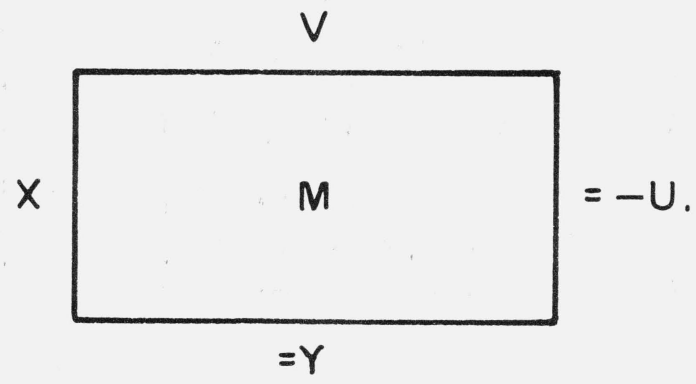

A simple pivot or $S$-pivot in (the tableau of) the dual linear systems $X M=Y$ and $M V=-U$ is a simultaneous exchange of variables $x_{i}$ with $y_{j}$ and $u_{i}$ with $v_{j}$, provided $m_{i j} \neq 0$, while leaving the remaining variables fixed.

Schematically, for $p=m_{i j} \neq 0$ :

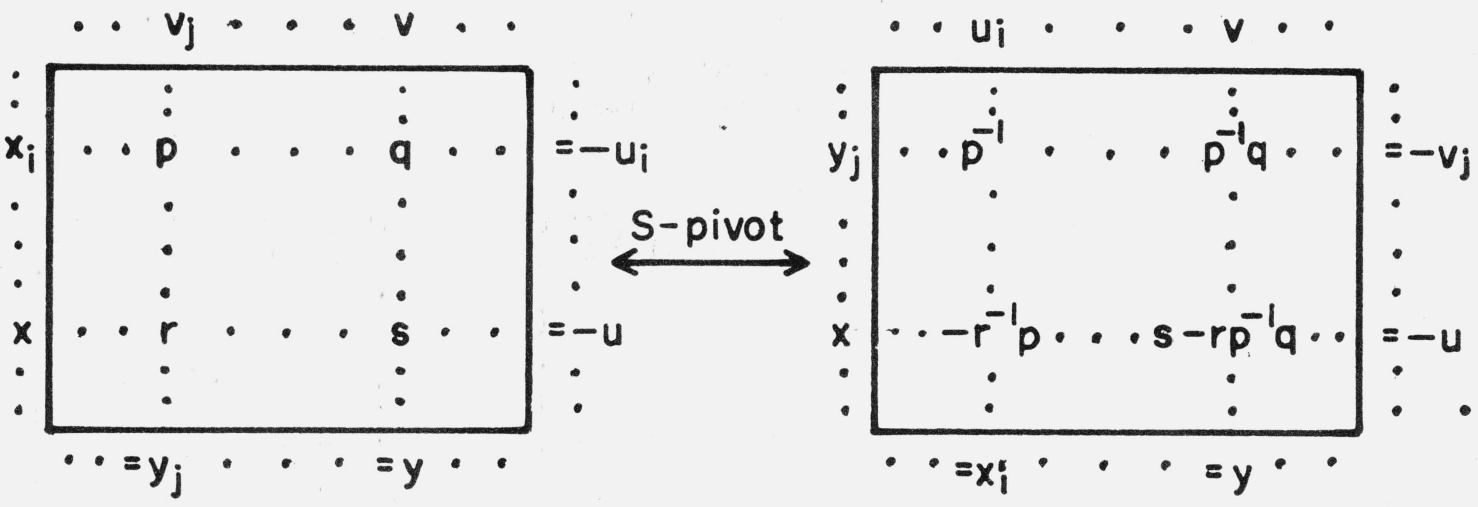

THEOREM 7: Given any rectangular matrix $\mathbf{M}$ over an ordered field, by a finite number of simple pivots in $\mathrm{M}$ we can reach a matrix $\overline{\mathrm{M}}$ having either a nonnegative row and a nonpositive row, or a nonnegative column and a nonpositive column, or a row and column of the same sign.

Schematically, Theorem 7 says we can reach one of the following:

(1)

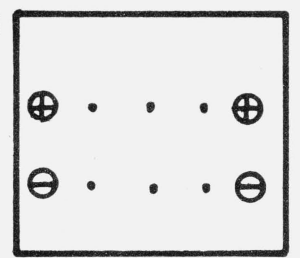

(2)

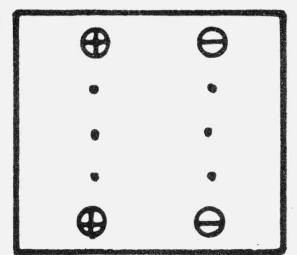

(3)

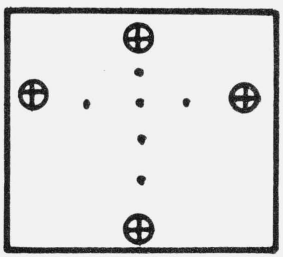

(4)

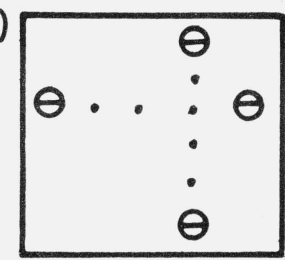

Note that the presence of a trivial row or column immediately gives us (1) or (2).

Proof: Given $M$, form an expanded skew tableau with $-M^{T}$ and blocks of zeros:
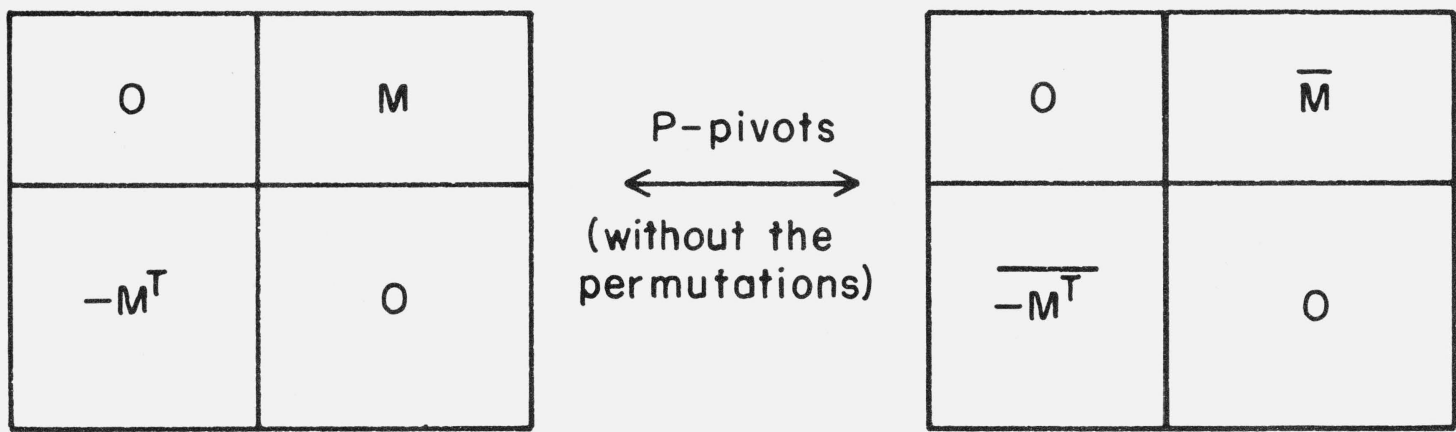
If $M$ has only one row or column, it is already in form (1), (2), (3) or (4), so assume $M$ has at least two rows and two columns.

By Theorem 1, a finite sequence of $P$-pivots in the expanded tableau yields a type (1) saddlepoint. If for each $P$-pivot we perform the inverse permutation, then $\bar{M}$ will remain in the upper right-hand corner, as though the $P$-pivot did not include a permutation. Such modified $P$-pivots correspond to pairs of $S$-pivots on $m_{i j}$ in $M$ and $-m_{j i}$ in $-M^{T}$. Moreover, pivots in $M$ or in $-M^{T}$ preserve the blocks of zeros. Hence by Theorem 5 we can repeatedly $P$-pivot in the expanded tableau ( $S$-pivot in $M,-M^{T}$ ) to reach a double saddlepoint.

If both rows of opposite sign appear in $\bar{M}$, then we have form (1) in $\bar{M}$. If both rows appear in $\overline{-M^{T}}$, then we have reached (2) in $\bar{M}$. One row in $\bar{M}$ and the other in $\overline{-M^{T}}$ yields (3) or (4) in $\bar{M}$, proving the theorem.

From its form it appears that Theorem 7 may lead to further results in the theories of positive semidefinite quadratic programs and of bimatrix games.

\section{References}

[1] Stein, Marjorie L., A pivot theory of skew matrices with applications, Doctoral Dissertation, Department of Mathematics, Princeton University, Princeton, N.J., 1972.

[2] Stein, Marjorie L., A saddlepoint theorem for self-dual linear systems, Technical Summary Report \#1311, Mathematics Research Center, University of Wisconsin, Madison, Wisconsin, 1973. To appear in Linear Algebra and Its Applications.

[3] Stein, Marjorie L., A double saddlepoint conjecture for skew matrices, Technical Summary Report \# 1315, Mathematics Research Center, University of Wisconsin, Madison, Wisconsin, 1973, in Transactions of the Nineteenth Conference of Army Mathematicians, Part 2, Report No. 73-3 (Durham, N.C.: U.S. Army Research Office, 1973), pp. $1073-1088$.

[4] Tucker, A. W., A combinatorial equivalence of matrices, in Proceedings of Symposia in Applied Mathematics, Vol. 10, edited by R. Bellman and M. Hall (Providence, R.I.: Amer. Math. Soc. 1960), pp. 129-140.

[5] Tucker, A. W., Combinatorial theory underlying linear programs, in Recent Advances in Mathematical Programming, edited by R. L. Graves and P. Wolfe (New York: McGraw-Hill 1963), pp. 1-16.

[6] Tucker, A. W., Pivotal Algebra, Lecture Notes by T. D. Parsons, Department of Mathematics, Princeton University, Princeton, N.J., 1965.

(Paper 78B4-412) 